\title{
Quantum efficiency of Pd/TiO 2 catalyst for photocatalytic reforming of methanol in ultra violet region
}

\begin{abstract}
The efficiency of $\mathrm{Pd} / \mathrm{TiO} 2$ catalyst for photocatalytic reforming of methanol was investigated in ultra violet, UV region by monitoring the volume of $\mathrm{H} 2$ production and the number of incident photon using chemical actinometry method. 2-nitrobenzaldehyde solution was used to evaluate the number of incident photon from solar light by monitoring the rate of photochemical isomerisation to 2-nitrobenzoic acid. The activity of $\mathrm{Pd} / \mathrm{TiO} 2$ catalyst varies across UV region with the ability to utilise the incident photon for $\mathrm{H} 2$ gas production is higher in UV-B. The quantum efficiency of the reaction was affected by the amount of catalysts concentration and the number of incident photons.
\end{abstract}

Keyword: Photocatalysis; Hydrogen; Pd/TiO2; Quantum efficiency; Chemical actinometry 